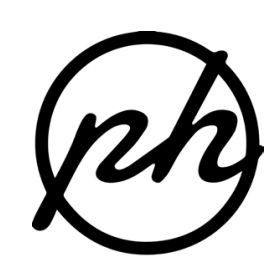

\title{
ESTUDIOS LINGÜÍSTICOS
}

\section{LOS ESTUDIOS ÁRABES EN LA CIUDAD DE SEVILLA Y EN SU UNIVERSIDAD: UNA APROXIMACIÓN A SU LARGA HISTORIA (SIGLOS XIII-XXI)}

\author{
ARABIC STUDIES IN THE CITY OF SEVILLE AND ITS UNIVERSITY: AN APPROACH TO \\ THEIR LONG HISTORY $\left(13^{\mathrm{TH}}-2 \mathrm{1}^{\mathrm{ST}}\right.$ CENTURIES)
}

\author{
Ana María Cabo GonzÁlez \\ ÁlVARO SuÁrEz ORTIZ \\ Universidad de Sevilla \\ acabo@us.es \\ suarezortizalvaro@gmail.com
}

Recibido: 08-12-2017

Aceptado: 02-03-2018

\section{RESUMEN}

Este trabajo constituye un estudio de naturaleza histórica y proyección diacrónica de la evolución de los estudios de lengua y cultura árabes llevados a cabo en Sevilla y su Universidad. Tras la conquista de la ciudad en 1248, Alfonso X funda aquí el primer centro dedicado a la enseñanza y el aprendizaje de la lengua árabe: el Estudio General de Latino y Arábigo. Tras la venida a menos de este primer centro, la Orden Franciscana de Frailes Menores crea en el siglo XVII el Colegio Trilingüe de Sevilla, donde destacaría Francisco Bernardino González, cuyas obras Intérprete Arábigo y Epítome de la Gramática Arábiga constituyen un excepcional modelo de diccionario y de gramática para el aprendizaje del árabe. En el siglo XIX, Pascual de Gayangos se convierte en uno de los eruditos más prolijos de nuestras letras, y se consolida como el padre el arabismo español. Entre sus discípulos hay que destacar a León Carbonero y Sol, figura clave de los estudios árabes en la Universidad de Sevilla. El siglo XX y el XXI culminan con la implantación definitiva de los estudios árabes en esta Universidad.

Palabras clave: Colegio Trilingüe de Sevilla; Estudio General de Latino y Arábigo; Francisco Bernardino González; León Carbonero y Sol; Pascual de Gayangos; Universidad de Sevilla.

\begin{abstract}
This work constitutes a historical-natured, diachronically projected survey of the evolution of the studies of the Arab people's language and culture carried out in Seville and its University. After the conquest of the city in 1248, Alfonso X The Wise founds herein the first center dedicated to the teaching and learning of Arabic: the Estudio General de Latino y Arábigo (General Study of Latin and Arabic). After the failure of this first center, the Franciscan Order of Friars Minor creates in the
\end{abstract}


$17^{\text {th }}$ century the Colegio Trilingüe (Trilingual School) of Seville, where Francisco Bernardino González would stand out as the author of Intérprete Arábigo (Arabic Interpreter) and Epitome de la Gramática Arábiga (Epitome of Arabic Grammar), both remarkable models of a dictionary and a grammar for learning Arabic. In the $19^{\text {th }}$ century, Pascual de Gayangos becomes the leading exponent of the Arabic letters of his time and educates the next generation of Arabists, including disciple León Carbonero y Sol, a key figure in Arabic studies at the University of Seville. The $20^{\text {th }}$ and $21^{\text {st }}$ centuries culminate with the final implementation of Arabic studies in this University.

Keywords: Colegio Trilingüe (Trilingual School) of Seville; Estudio General de Latino y Arábigo (General Study of Latin and Arabic); Francisco Bernardino González; León Carbonero y Sol; Pascual de Gayangos; University of Seville.

\section{INTRODUCCIÓN}

En el año 1248 Sevilla (Išbiliyya) deja de ser musulmana y se incorpora a los dominios de la cristiandad tras un largo asedio de dieciséis meses que concluía con la capitulación y la entrega de las llaves de la ciudad al rey Fernando III el Santo, quien haría ondear el pendón real de la Corona de Castilla desde los muros del alcázar, el día 23 de noviembre del mencionado año (Bosch 1988; Ladero 1989: 1518), marcando de tal modo el surgimiento de la Sevilla cristiana, conformada a lo largo de los siglos en los derroteros de un devenir histórico que ha dado lugar a la ciudad que conocemos en la actualidad.

Entre la génesis de la ciudad de Sevilla como urbe castellana y el surgimiento de las primeras instituciones docentes que dieran comienzo al estudio del árabe dentro de sus muros no transcurrió ni una década. Los primeros atisbos del estudio de la lengua así como el establecimiento de la que puede considerarse la primera institución de enseñanza dedicada a su conocimiento se derivan de la ingente labor intelectual llevada a cabo por el rey Alfonso X el Sabio, hijo y heredero del conquistador de la ciudad.

Eloy Benito Ruano (2000, Tópicos: 100) señala que ya desde el siglo X existía un interés en los diferentes reinos cristianos peninsulares por el estudio de manuscritos árabes de diversa temática, circunstancia que trajo aparejada la producción de un importante volumen de traducciones de obras de matemáticas, medicina y astronomía al latín (Haskins 1924; Millás 1942; Millás 1954: 395-428; Thorndike y Kibré 1963; Vernet 1978; Scarcia 1987; Burnett 1992: 1036-1058; D’Alverny, La connaisance 1994; D’Alverny, La transmission 1994; Burnett 1995: 214-235). De igual modo, se concedió especial relevancia al conocimiento de la lengua árabe y de la cultura islámica, con el fin de lograr la conversión de la población musulmana que residía en tierras cristianas. No obstante, fue durante el reinado de Alfonso X cuando el interés por las ciencias y la literatura andalusíes alcanzaron su apogeo (Millás 1933: 155-187; Procter 1945: 155-187; Procter 1951: 12-29; Samsó 1997, vol. I: 199231; Benito 2000, “Ámbito": 13-28). Diversos centros de enseñanza vieron la luz en 
distintas ciudades españolas bajo los auspicios del rey sabio, como la Escuela de Murcia (fundada durante su reinado y dirigida por el filósofo al-Riquti) o el Studium salmantino, cuyo funcionamiento reguló en su libro de Las Siete Partidas, y al cual dotó de refrendo real en 1248, tres décadas después de su establecimiento.

\section{LOS ORÍGENES: EL ESTUDIO GENERAL DE LATINO Y ARÁBIGO}

El día 28 de diciembre de 1254, tan solo seis años después de la conquista de Sevilla a los musulmanes, Alfonso X, que por aquel entonces llevaba tres años ocupando el trono de Castilla, otorga a la ciudad un privilegio rodado, el más solemne de los documentos emitidos por la cancillería real castellana (Borrero 1955: 224-225), mediante el cual establece su voluntad de fundar una institución que llegase a operar como un estudio e escuelas generales de latino e de aráuigo. Este centro, que contaba con categoría de Estudio General, fue la primera institución que incluyó estudios sobre la lengua árabe en Sevilla, e incluso podría haberse instituido como el embrión de la Universidad actual, de no haber sido por su efímera existencia y su pronto relegamiento a la categoría de Estudio Particular, circunstancia que aceleró su desaparición.

La escasa trascendencia del Estudio General de Latino y Arábigo en el conjunto de la historia de la los estudios árabes sevillanos y lo efímero de su duración pueden entenderse como las causas que expliquen la escasa bibliografía que se ha dedicado a describir su funcionamiento o a aportar cualquier otra clase de dato que permita esclarecer las circunstancias justificadoras de su incapacidad para seguir en activo con el paso del tiempo. Varios investigadores aducen las razones que probablemente motivaran al rey sabio a fundar la institución, en las que se aprecia un claro interés social. Así pues, J. A. Carrillo Salcedo (2005: 10) señala que la concesión de tal privilegio ennoblecía e incrementaba el prestigio de una ciudad que ya gozaba de una cierta trascendencia (había sido tanto la capital de la taifa de Sevilla como la de los dominios almorávide y almohade en la península ibérica). Además, se hacía necesario contar con eclesiásticos e intérpretes que pudieran hacer de mediadores entre esta nueva comunidad cristiana y los grupos mudéjares que continuaban residiendo en la ciudad y en el campo de la zona recién conquistada. Igualmente, M. González Jiménez (1998: 52) también apunta la idoneidad de la institución para el desarrollo del programa de traducciones que auspiciaba el monarca castellano a través de sus famosos trabajos en la Escuela de Traductores de Toledo. Por su parte, J. Sánchez Herrero (1984: 876), basándose en las reflexiones del padre Denifle y de Cándido M. Ajo González de Rapariegos, opina que este estudio no tenía finalidad religiosa, sino científica y que, pese a recogerse en su carta fundacional las lenguas latina y árabe como único objeto de estudio, no se limitó a ellas, sino que albergó también a estudiantes de medicina, que se hospedaban en un edificio cercano

1 Véase La primera etapa de la Universidad de Sevilla. <http://personal.us.es/alporu/historia/ historia_1.htm\#h1_2_1/ > . 
habilitado a tal efecto, pues, como señala M. I. González Ferrín (1999: 264), al estudio pueden acudir escolares de todo el reino sin necesidad de pagar portazgo por los libros y cosas personales que trajesen. Esta idea también es respaldada por E. Benito Ruano (200o, Tópicos: 100), quien afirma que Alfonso X se trasladaría a Sevilla para crear un Estudio General de latín y árabe con maestros musulmanes para enseñar medicina y otras ciencias.

El Estudio de Sevilla fue elevado a la categoría de Estudio General en 126o de forma oficial, a través de una bula papal emitida por Alejandro IV el 30 de junio, que permitía a los docentes y estudiantes de la institución mientras permanezcan ocupados en sus estudios, [percibir] los frutos de sus prebendas y beneficios no curados, como los perciben los residentes en los demás estudios generales (Sánchez Herrero 1984: 876), satisfaciendo de ese modo las pretensiones con las que Alfonso X emprendió su fundación. No obstante, el rey castellano no le asignó nunca dotación alguna, hecho que, sumado al escaso apoyo brindado por Sancho IV, desembocó en la pérdida de su estatus, siendo relegado, a efectos prácticos, al nivel de Estudio Particular (instituciones de menor rango que no eran creadas por el rey ni por el Papa, sino por un particular o un ayuntamiento), y eventualmente a su desaparición.

\section{El Colegio trilingüE de SeVilla}

\subsection{Antecedentes}

El hueco dejado tras la venida a menos del Estudio General fundado por Alfonso $\mathrm{X}$ no tardaría en ser ocupado por la contribución que otros hombres de letras realizarían en pro del cultivo de la lengua árabe. En el año 1209, treinta y nueve años antes de la conquista de Sevilla, el santo italiano Francisco de Asís funda la Orden de Frailes Menores, cuyo interés y compromiso para con la lengua árabe sería el desencadenante de una secuencia de acontecimientos que terminarían por dar lugar a la fundación, tres siglos después, de otra institución que se dedicó al cultivo y a la enseñanza del árabe en la ciudad de Sevilla: el llamado Colegio Trilingüe ("El estudio", Lourido 2006: 9-240).

A. Bueno García resume en las siguientes líneas el acontecimiento que llevaría a marcar de forma ineludible la vinculación de la orden de San Francisco de Asís con la lengua árabe:

Todo empezó sin duda en los albores del siglo XIII, cuando San Francisco, guiado por su celo de conocer y afianzarse en tierra infiel, arribó a ella con un grupo de hermanos y, fruto del encuentro en [D]Amieta con el mismísimo sultán Malek-el-Kamel, se forjaron unas relaciones de confianza que posibilitaron el establecimiento con el tiempo de una presencia en Tierra Santa y en zonas del norte de África que dura hasta nuestros días. (Bueno 2013: 91). 
La documentación consultada señala que esta primera toma de contacto franciscana en Tierra Santa y en otros lugares con población de lengua árabe y cultura islámica parece responder a la voluntad de consolidar, en dichos territorios, una presencia cristiana que no se mostrase provocadora o belicosa, sino que acudiese con ánimo de integrarse y convivir con la sociedad autóctona desde el respeto hacia su religión, en aras de lo cual llegaron a adoptarse severas medidas que prohibían a los franciscanos predicar, y contemplaban la expulsión e incluso el martirio de aquellos que las incumpliesen, pretendiéndose en su lugar que los franciscanos se acercaran a los musulmanes, dando ejemplo de vida a través de sus acciones y testimonios (Bueno 2013: 91-92).

En 1342, la promulgación por parte del papa Clemente VI de las bulas Gratias agimus y Nuper carissimae oficializa definitivamente la presencia franciscana en Tierra Santa, al designar a la orden como custodia de los Santos Lugares (Bueno 2013: 91). La garantía de la misión acometida por los franciscanos y el éxito en su acercamiento a los habitantes de la zona repercutía, en gran medida, en la adecuada formación de los misioneros interesados en acudir a Tierra Santa, para quienes el conocimiento de las lenguas habladas en Oriente Próximo constituía un requisito que les posibilitaría ejercer dignamente su labor evangélica entre los autóctonos de aquellas regiones (Bernardino 2005: 45). De tal modo, como señala Ramón Lourido Díaz, se hizo necesaria la creación de centros docentes, Estudios y Colegios, en los que los futuros misioneros franciscanos pudieran aprender a comunicarse eficientemente con la heterogénea población de Tierra Santa, compuesta por judíos, musulmanes y cristianos que tenían al hebreo, al árabe o al griego como lenguas maternas.

El primer centro de estas características que vio la luz en el actual territorio español fue el Colegio Orientalista y Misional de Miramar, fundado por el célebre filósofo y místico Ramón Llull en 1276, bajo los auspicios de Jaime II y del papa Juan XXI. Durante sus diecisiete años de actividad reunió en sus dependencias a trece franciscanos dedicados al estudio de la lengua árabe (Bueno 2013: 92-93).

Desde que en el Colegio Orientalista y Misional de Miramar decayeran sus actividades sobre el estudio de la lengua árabe, no encontramos en nuestra historia otro hito significativo hasta finales del siglo XV, concretamente en el año 1470, cuando nace en Sevilla Fernando de Contreras, que fue ordenado sacerdote y, más tarde, en 1515, nombrado Capellán Mayor del Colegio de San Ildefonso de Alcalá de Henares. El Venerable Contreras, como es conocido, se distinguió por su intensa actividad en la redención de cautivos cristianos que se encontraban en manos de musulmanes en el norte de África, actividad que llevó a cabo hasta su muerte en $1548^{2}$. Su extraordinario conocimiento de la lengua árabe hablada por los

\footnotetext{
2 Sobre la vida de este singular sevillano, véase Gabriel de Aranda, Vida del siervo de Dios exemplar de sacerdotes el venerable padre Fernando de Contreras natural de esta ciudad de Sevilla, Sevilla, 1698. 
musulmanes norteafricanos y su intensa labor diplomática le valió la redención de un gran número de cristianos apresados y cautivos.

Si bien hubo intenciones manifiestas de crear nuevas instituciones de estudio de lenguas orientales en el territorio peninsular en repetidas ocasiones, la idea no terminó de madurar y llevarse a término hasta bien entrado el siglo XVII (Lourido, El estudio 2006), cuando el padre Juan Albín, ministro general de la Orden de los Frailes Menores, resolvió habilitar el convento de San Francisco de Sevilla para la instauración de un nuevo centro de estudio franciscano, que vino a ser conocido bajo la denominación de Colegio Trilingüe de Sevilla (Bernardino 2005: 48). La escasez bibliográfica no permite establecer con exactitud las causas que motivaron al padre Albín a inaugurar el Colegio Trilingüe en Sevilla, aunque Ramón Lourido Díaz hace mención en su obra de un documento que puede arrojar algo de luz sobre las causas subyacentes a la fundación del centro. Se trata de una Relación de las Misiones de la Custodia de Tierra Santa, que recoge las impresiones del padre Arguimbau tras su visita a los religiosos de la Custodia de Tierra Santa entre los años 1691 y 1692, la cual tenía por objeto comprobar el estado en el que se hallaba dicha Custodia en aquel entonces, y cómo procedía su labor evangelizadora. Lo reflejado por el padre Argimbau en su Relación lleva a Ramón Lourido Díaz a pensar que el padre Albín, sabedor del deficiente conocimiento de las lenguas de aquellas regiones entre los religiosos de Tierra Santa [...] terminó optando por la fundación de la Escuela Trilingüe de Sevilla (Bernardino 2005: 49). Dos son las circunstancias que nos permiten llegar a esa conclusión. En primer lugar, la inspección llevada a cabo en los territorios de Egipto, Constantinopla, Siria y Palestina, que fue completada con la correspondencia que el franciscano mantuvo con varios frailes residentes en Tierra Santa, permite afirmar que el conocimiento lingüístico de los misioneros era, cuanto menos, mediocre, tal y como lo describe el propio Arguimbau: “[... en Sayda, Acre y Nazaret no había quien supiese la lengua árabe» / fol. 215 v/; [...] no había en Damasco religioso alguno que estudiase la lengua áraba, y en Alepo me dijeron que no había más que uno /fol. 214 v/; [...] que, finalmente en Ariza [Harisa], no había ni religiosos que estudiasen la lengua áraba /fol. 202 v/ (Lourido 2001: 149)".

Por otro lado, la deficiente labor llevada a cabo en la mencionada ciudad de Harisa, donde Francisco de Jesús María y José León habían fundado un seminario árabo, había llegado a los oídos del padre Albín y de Arguimbau, quien pudo corroborar la veracidad de dicha situación a través de las cartas que intercambió con Rafael Ventayol y Francisco Jordán, dando cuenta de la misma en su Relación en los siguientes términos:

/fol. 214 r/ Informado de este punto, he entendido que el seminario del AnteLíbano, que llaman de Ariza [Harisa], siempre ha sido y es de ningún provecho, y por esto ha habido siempre controversias en su fundación, y ahora los superiores no hallan religioso alguno que quiera estar en él (sino es el fundador), ni conviene mudarle en otra parte del monte Líbano, porque los maronitas que habitan en él 
están bien asistidos del patriarca, obispos y curas, los cuales no quieren que los misioneros [franciscanos] confiesen sus parroquianos, y no ha mucho tiempo que el patriarca de los maronitas y un obispo excomulgaron los misioneros porque confesaban, y sus feligreses que se confesaban con ellos (Bernardino 2005:50-51).

Los datos que nos proporciona Ramón Lourido Díaz nos permiten elucubrar, sin mucho desatino, que, tanto la baja competencia lingüística de los franciscanos al servicio de la Custodia de Tierra Santa como la dificultad de subsanar ese déficit de conocimiento exclusivamente in situ mediante los centros allí establecidos, fueran los detonantes que movieran al padre Albín a concebir un nuevo proyecto de enseñanza que permitiera instruir, desde España, a los misioneros por algún tiempo en la lengua arábiga y griega y, después, pasar a la Sancta ciudad de Jerusalén, y recibir del Guardián y Custodio las asignaciones convenientes... (Bueno 2013: 93). Bajo esta premisa nacía el Colegio Trilingüe de Sevilla en el año 1694.

\subsection{Documento fundacional y elección de la sede}

El primer documento alusivo a la existencia del Colegio Trilingüe del que se tiene constancia viene constituido por un breve párrafo, redactado por el padre Fernando Valderrama, que reza:

El año 1694 fue instituido este convento [San Francisco de Sevilla] por una de las tres casas destinadas para el estudio de las tres lenguas, Hebrea, Griega y Arábiga, asignando para este fin tres Lectores y doce estudiantes. Debía dar lección todo el año, a excepción de los meses de Julio y Agosto, y en cada día tres horas, una respectiva a cada Lector. Tienen estos mismos las preeminencias que tienen los Lectores de Teología, pero en el asiento eran inferiores a éstos. Los estudiantes debían ser predicadores nombrados por el Ministro General de la Orden para este estudio, y podían ser de ésta o de otra Provincia. Todo esto consta de Patente original del Rvdmo. Fr. Buenaventura Povio, Ministro General de la Orden, su data a 9 de Agosto del expresado año. Hallábase ésta entre otros papeles de la Librería del Convento. Cesó este estudio, pero se ignora el Motivo ${ }^{3}$.

Ramón Lourido Díaz (Bernardino 2005: 56-59) efectúa un minucioso análisis del párrafo reproducido que nos permite extraer una serie de conclusiones en torno a los primeros momentos de la institución de lenguas. El autor hace alusión al desconocimiento que Valderrama debía tener acerca del funcionamiento y gestión interna del centro y del modo en el que la docencia estaba organizada en el mismo, ya que en su carta se limita a mencionar que terminó por cerrar, pero no da cuenta de los motivos que ocasionaron el cese de su actividad. Asimismo, arguye que es bastante probable que una de las tres escuelas que el padre Valderrama cita en su carta se corresponda con el Convento Recoleto del Valle, ubicado, como el Convento

3 Extraído de: Centuria Bética-Convento y casa grande de San Francisco de Sevilla. Sevilla: Archivo de la Provincia Franciscana Bética (APFB). Fol. 54-55.

ISSN 1132-0265

http://dx.doi.org/10.12795/PH.2017.i31.o1

Philologia Hispalensis 31/1 (2017) 11-36 
de San Francisco de Sevilla, en la ciudad homónima. Basándose en los ejemplares de la Patente de fundación del centro (mencionada en el párrafo de Valderrama) que tiene a su disposición, el investigador señala que [el Convento Recoleto del Valle] era el convento elegido para cobijar el Colegio de Lenguas en Sevilla (Bernardino 2005: 57), hipótesis que discutiremos posteriormente. Por último, recalca el error cometido por el padre Valderrama al atribuir a Buenaventura Poerio (a quien erróneamente cita en su carta como "Povio") la autoría de la mencionada Patente del Colegio Trilingüe, aportando pruebas documentales que confirman que el texto fundacional fue obra del padre Juan Albín, quien lo redactó poco tiempo antes de terminar sus funciones como ministro general de la Orden, y la difundió entre las diferentes Provincias Franciscanas de la Observancia de España para su compilación en el Libro de Patentes de todos los conventos franciscanos diseminados por el país (Bernardino 2005: 57-59).

Tanto el padre Valderrama como el padre Albín dan constancia en sus respectivos escritos de que el lugar designado para el emplazamiento del Colegio de Lenguas se correspondía con el Convento del Valle, si bien, pruebas documentales posteriores confirman que fue efectivamente el Convento de San Francisco de Sevilla el edificio que albergó la sede de la institución. Las investigaciones de Ramón Lourido Díaz (Bernardino 2005: 62-63) sugieren que la consideración del primero de los dos conventos respondería a la premura en abrir el Colegio de Lenguas lo antes posible, [de ahí que] se indicase a los religiosos de otras Provincias un lugar seguro adonde acudir [...] provisionalmente, aunque, en avances posteriores, descarta directamente que dicho edificio llegase a funcionar como sede en ningún momento, ya que el mismo padre Albín emitió, a través de un intermediario, un mandato, poco después de firmar la Patente, en el que manifestaba su deseo de que el colegio fuera instalado en uno de los centros de su jurisdicción, indicando que muy al propósito se disponga dicho Colegio en este convento de N.P. San Francisco Casagrande de Sevilla. La aceptación de este mandato por parte de las autoridades de la Provincia Franciscana de Andalucía refuerza la suposición de que el Convento del Valle no llegó ni siquiera a comenzar las clases de lenguas.

A modo de conclusión, cabe señalar que la aprobación del proyecto del padre Albín y la puesta en marcha del Colegio Trilingüe tuvo como consecuencia inmediata la obligatoriedad de la formación en lenguas orientales de todos aquellos que, en lo sucesivo, deseasen embarcarse hacia Tierra Santa y desempeñar su cometido entre los infieles. No obstante, la inscripción en el centro estaba sujeta a una serie de requisitos previos de índole tanto personal como profesional, que Antonio Bueno García y Ramón Lourido Díaz recogen en sus páginas. Así, en cuanto al primer requisito, era necesario que el perfil de los aspirantes se correspondiera con el de una persona de ánimo sosegado, de índole dócil y puras costumbres, y estar dotado de suficiente doctrina para poder imbuir aquellas gentes en la Palabra de Dios $y$ en los dogmas de la fe (Bueno 2013: 93). A esta misión para con los habitantes autóctonos se sumaba el ministerio pastoral que los misioneros igualmente habrían ISSN $1132-0265$ 
de desempeñar para alimentar la fe de los cristianos residentes en aquellos lugares, motivo de más que requería una nobleza de espíritu como la que el padre Ventayol describía en una de sus cartas:

...la Custodia [de Tierra Santa] necesita obreros y ministros en lengua arábiga y griega, y por lo tanto aga proveer una docena de Religiosos moços, o por lo menos siete u ocho, que sean de santas costumbres y buen natural, y que no sean caprichosos sino muy mansuetos, porque en el aprender las lenguas y después en el exercicio de los Officios que se han de tener y es menester resistan a muchos travajos y adversidades, y si son poco pacientes y caprichosos no resisten ni prueban bien y dan muchos escollos, como bien le informará el Comissario Martín de lo que passa por aca, y que traigan orden de entrar derechamente a la

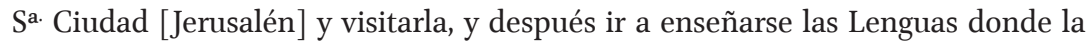
obediencia les mandare, y el tiempo que abian de estar en el Colegio de Sebilla, aquí se aprovechará mejor, y serán hábiles para administrar los sacramenteos y entre tanto en el dicho Collegio se pueden enviar otros... (Bernardino 2005: 87).

El segundo requisito, como señala Ramón Lourido Díaz (Bernardino 2005: 63), solamente posibilitaba el ingreso al Colegio de sacerdotes, ya que se requería que los interesados en estudiar en sus aulas hubieran previamente hecho promesa de pasar a Tierra Santa, estuvieran instituidos como Lectores, Predicadores o Confesores de seglares y no rebasasen los 40 años de edad.

\subsection{El Colegio Trilingüe en el Convento de San Francisco de Sevilla}

Como se ha mencionado, fue el propio padre Juan Albín quien seleccionó por mandato el Convento de San Francisco para instalar el Colegio de Lenguas. Para ello, encargó a Fr. Andrés de Barrera, residente de dicho convento y por aquel entonces vicecomisario de Tierra Santa ante la Provincia Franciscana de Andalucía, que remitiese al Provincial y su Definitorio o Consejo, una serie de escritos en los que se procediera a decidir, junto con la elección del convento, las dependencias que del mismo se destinarían a la impartición de docencia, y las obras y reestructuraciones necesarias para adecuar el edificio a tal fin (Bernardino 2005: 65-72).

La correspondencia entre Barrera y el Definitorio revela que la negociación entre ambas partes culminó con la cesión de la parte del edificio que la historiadora María José del Castillo Utrilla identificó, tras reconstruir el plano del convento, con la llamada hospedería de Indias, a la que describe como espaciosa, de dos pisos y patio con claustrillo en uno de sus lados, fuente y banco de azulejos. Toda ella estaba encalada y sus cubiertas eran de madera. El patio, al igual que las demás estancias, estaba enlosado y tenía zócalos de alizanes (del Castillo 1983: 393-398; del Castillo 1998: 94). El espacio destinado tenía capacidad suficiente como para incluir las quince salas solicitadas, tres de las cuales serían para uso personal de los Padres Lectores, y las doce restantes para los alumnos y la impartición de docencia. Una vez concluidas las obras necesarias para remodelar y adaptar las diferentes estancias, el 
Colegio Trilingüe abrió sus puertas para la enseñanza del griego, el árabe y el hebreo en una fecha cercana a mayo de 1695 .

\subsection{Profesores de lengua árabe y sus alumnos en el Colegio Trilingüe}

El día 29 de mayo de 1694 el padre Buenaventura Poerio de Taberna sucedió a Juan Albín en el cargo de Ministro General, demostrando el mismo interés por la formación de los religiosos franciscanos en lenguas orientales que había caracterizado a su predecesor. Así, pocos meses después de la promulgación de la Patente Fundacional del Colegio Trilingüe, el nuevo ministro realizó un segundo llamamiento para que misioneros franciscanos, tanto de las Provincias españolas como de otras instituciones europeas (como la de S. Pedro in Montorio, en Roma), acudieran a instruirse en sus aulas. Uno de los primeros en responder a la llamada fue el padre Francisco Bernardino González (2005: 73-76).

Ramón Lourido Díaz refiere también el nombre de dos profesores que impartieron lengua árabe en el centro, pero la reducida documentación de que dispone solo le permite aportar una concisa descripción general de su persona. El primero de ellos, de nombre Fr. Buenaventura da Molazzana, fue un franciscano italiano que, tras obtener un sólido conocimiento del idioma gracias a su trabajo como misionero en Egipto y en zonas de Siria y Palestina, fue convocado para ejercer de profesor en el Colegio de San Pedro in Montorio, de donde pasó a ocupar el mismo cargo en el Colegio de Sevilla a finales del año 1693. Por desgracia, no se dispone de documentación adicional que permita conocer detalles de la labor desempeñada en el centro. El otro profesor del Colegio sevillano fue Fr. José de León. Formado probablemente en la Escuela que los franciscanos habían fundado en Damasco, obtuvo el título de Lector de lengua árabe en 1681. Tras enseñar en la casa de Harisa (Líbano) y prestar sus servicios como secretario general en la Curia de los Frailes Menores, se trasladó a Sevilla para ejercer como profesor en el Colegio Trilingüe desde las fechas más tempranas de su apertura, teniendo como alumno al ya citado Bernardino González, cuya figura analizaremos con profusión más adelante.

En cuanto al alumnado que frecuentó las aulas del centro, cuya figura más destacada ya hemos mencionado, Ramón Lourido Díaz (Bernardino 2005: 84) proporciona una lista de nombres, y señala que los múltiples llamamientos para engrosar las listas de pupilos en el Colegio Trilingüe recibió una respuesta no muy entusiasta por parte de los llamados, bien que [...] en 1714, todavía algunos religiosos seguían pidiendo ir a Tierra Santa y estudiar las lenguas orientales, debido a que el interés de los susodichos radicaba más en poder servir como custodios de los Santos Lugares en los que Jesucristo había vivido y predicado su doctrina (tarea que no requería necesariamente el conocimiento de ninguna lengua autóctona), que en el deseo de evangelizar a la población no cristiana de la zona. 


\subsection{Período de actividad del Colegio}

Como ya apuntara el padre Valderrama, el Colegio Trilingüe de Sevilla tuvo una vida corta, y se dispone de muy escaso material que dé cuenta del período de tiempo durante el cual se extendió su actividad docente. Ramón Lourido Díaz recoge algunos datos que permiten afirmar que aún seguía en activo cinco años después de que el padre Bernardino González pasara por sus aulas entre los años 1694 y 1696, pues en 1701 se tiene constancia de que el padre Ventayol envió desde Tierra Santa sendas cartas al Ministro General de la Orden y al padre Comisario de Madrid en las que, respectivamente, proponía a Martín de Santander como profesor de griego en el Collegio de Sebilla, haziendole Lector de dicha lengua (Bernardino 2005: 86), e informaba de la necesidad de contar en la Custodia con miembros bien formados en griego y en árabe para desempeñar un eficiente ministerio en los Santos Lugares, como ya ha quedado referido. No obstante, el autor maneja otras referencias que llevan a pensar que, para el año 1707, el Colegio sevillano ya habría cerrado sus puertas, pues en la correspondencia intercambiada entre Juan Gil y Andrés de la Barrera, quienes ostentaban los cargos de Ministro Provincial y Procurador de Tierra Santa en los tiempos de la fundación del Colegio, no hacen mención alguna al mismo, lo cual lleva a concluir que debía haber cesado su actividad docente por aquel entonces.

\subsection{El Padre Francisco Bernardino González y su aportación a los estudios de lengua árabe}

Fr. Bernardino González, alumno del Colegio Trilingüe de Sevilla, es la figura más representativa de las que albergara la mencionada institución en sus aulas, debido a la trascendencia que su labor en Tierra Santa tuvo para el arabismo español. Las enseñanzas que recibió durante sus dos años de estudiante en el centro sevillano dieron fruto, a lo largo de su posterior labor en Oriente Próximo, en forma de diversas obras que pasarían a constituirse como material obligatorio de instrucción y enseñanza de la lengua árabe, que vendría a ser utilizado por los misioneros que lo relevaron en la labor evangelizadora franciscana desempeñada en los Santos Lugares (Bernardino 2005: 104).

\subsubsection{Datos biográficos}

Abdelouahab El Imrani (1998: 144) señala algunos datos biográficos sobre el franciscano, que él mismo reflejó en las páginas de su Intérprete Arábigo, obra que examinaremos detenidamente en páginas posteriores. Antonio Bueno García (2013: 95-98) aporta también algunos detalles sobre su persona. Según esta información, Francisco Bernardino González, oriundo de Castilla, era un fraile menor de la Provincia Observante de la Purísima Concepción, probablemente nacido con el nombre laico de José Rufo, y que vivió entre los años 1650 y 1750. Pasó dos años en 
el Colegio Trilingüe de Sevilla antes de dirigirse a Tierra Santa, donde prosiguió y perfeccionó los estudios iniciados en la capital andaluza, y desempeñó los cargos de Lector de lengua árabe en el Colegio de Damasco, Guardián de Belén y Párroco de Rama. El mismo Francisco Bernardino González resume este período de su vida con las siguientes palabras:

En Damasco residí por tres veces enseñando por tres años la lengua árabe a los Religiosos, regentando el resto del tiempo el oficio de Guardián [superior] y Párroco, habiendo hecho lo mismo en el convento y ciudad de San Juan de Judea y en Rama. Por manera que mi permanencia en aquellas misiones duró el espacio de 16 años: tiempo suficiente para adquirir el uso, pronunciación y fuerza del idioma árabe generalmente adoptado en aquellas regiones (Bernardino 2005: 115).

El cometido que concentró parte de la atención de Bernardino durante su estancia en Tierra Santa fue la continuación de la labor iniciada en el Colegio de Sevilla por su maestro Fr. José de León, quien había empezado a acometer la ordenación de un diccionario árabe bilingüe. La compleción de esta diligencia dio lugar a la obra que pasamos a describir a continuación.

\subsubsection{Una obra clave: El Intérprete Arábigo (Asín 2007: 267-279)}

A. Bueno García (Bueno 2013: 98) señala que de la obra del P. Bernardino se encuentran diversos ejemplares, pero todos realizados por sus discípulos. El profesor vallisoletano refiere un repertorio de hasta siete copias distintas localizables dentro de España. A tenor de la bibliografía disponible consultada, se puede afirmar que el Intérprete de Bernardino González constaba de tres partes claramente diferenciadas: una castellano-árabe, otra árabe-latín y una tercera árabe-castellano (Bueno 2013: 95).

El cotejo documental efectuado por Abdelouahab El Imrani (1998: 134) y su escrutinio de los manuscritos a los que pudo acceder en la Biblioteca Islámica Félix María Pareja de Madrid y en la Biblioteca Universitaria de Leyden, nos brinda un apoyo excelente sobre el que referir nuestra propia descripción general del contenido del notable libro del que venimos ocupándonos, que se basará en la información contenida en el ejemplar reproducido por Juan Gallego, al que tanto Ramón Lourido Díaz como el propio Abdelouahab El Imrani recurrieron para dejar constancia de las características de la obra de González.

\subsubsection{Descripción general del Intérprete Arábigo}

Antes de acometer tal menester procedemos a dejar constancia del hallazgo de una anotación en la parte árabe-castellano de uno de los manuales de la Biblioteca Islámica Félix María Pareja, que no figura en todos los demás ejemplares conocidos del Intérprete. Se trata de la que parece ser una versión del himno latino Tota 
pulchra es, dedicado a la Virgen María. Reproducimos a continuación el texto de la anotación tal y como aparece en lengua árabe (El Imrani 1998: 139):

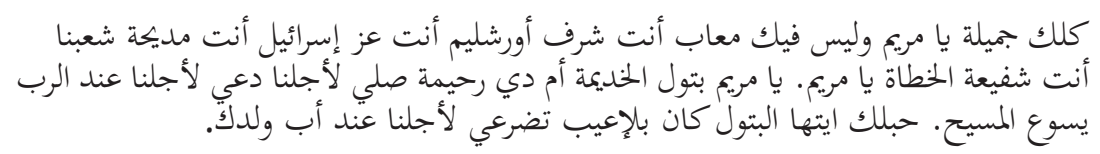

Para el que ofrecemos la siguiente traducción al español:

Toda eres hermosa, oh María, y no hay en ti mácula. Eres el honor de Jerusalén, eres la gloria de Israel. Eres la loada de nuestro pueblo. Eres la intercesora de los pecadores, oh María. Oh María Virgen, madre servidora y misericordiosa, reza por nosotros, ruega por nosotros ante el señor Jesucristo. Tu gravidez, oh Virgen, no tuvo tacha. Suplica por nosotros ante el padre de tu hijo.

Concluida esta breve digresión, retomamos la descripción del Intérprete, que iniciamos con la parte castellano-árabe del mismo, y que se referirá a la copia manuscrita de Juan Gallego. Con un total de cuatrocientas treinta y cinco páginas, incluye, en la primera de las hojas, el título completo de la obra, que reza como sigue:

\section{INTERPRETE ARABIGO CASTELLANO}

El qual en lengua araba da interpretada la castellana, que es la mas unibersal de España.

Compuesto, y ordenado por A P. F. Bernardino González, fraile menor, hijo de la Sta Prov. de la Puríssinia Concepción en España ex Guardian de Belem, Curato de Rama, y al presente, Curato, Vicepresidente, y Lector Arabo en la ciudad de Damasco y trasladado Por el Padre fr. Juan Gallego Hijo de la dicha Provincia (Missionario Apostolico y Colegial en dicho colegio de la ciudad de Damasco) [texto ilegible] y al presente estudiante Aravo en este colegio de Damasco (El Imrani 1998: 140-41).

El resto de la página está ocupado por un prólogo o proemio en el que el autor expone diversas cuestiones.

La segunda parte de la obra es la sección árabe-latín, de no más de una decena de páginas de extensión. Incluye un corto proemio en latín, y el léxico que recoge versa sobre términos philosophicos y theologicos que he podido acquirir no sin trabajo (El Imrani 1998: 141).

La tercera y última parte es la correspondiente a la parte árabe-español. De unas doscientas treinta hojas de extensión, incluye el proemio, en el que Fr. Bernardino hace patente su intención de ampliar y completar el diccionario que su maestro José de León iniciara en el Colegio Trilingüe de Sevilla (Bernardino 2005: 154-155). 
3.6.3 El Epítome de la Gramática Arábiga

Nos parece adecuado concluir este gran apartado sobre la figura y obra del sacerdote franciscano Bernardino González dedicando algunas líneas a la descripción de otra de sus grandes obras, en este caso de carácter gramatical: el llamado Epítome de la Gramática Arábiga.

De acuerdo con la información proporcionada por Antonio Bueno García (2010: 35 ), este compendio gramatical, al igual que el diccionario, tienen la particularidad de haber pasado en forma manuscrita, pues a pesar de ser aprobadas en vida del autor para pasar a la imprenta, nunca llegaron a la misma. Lo declarado por este autor entra en conflicto con la información contenida en el Boletín de la Real Academia de la Historia del año 1976, en el que se afirma que elP. Bernardino González imprimió en Madrid, 1719, su Epítome de la Gramática arábiga, y dejó manuscrito en Jerusalén [...] su grueso Diccionario Español-Árabe ${ }^{4}$.

En cualquier caso, es el profesor vallisoletano quien más información pone a nuestra disposición, y a quien nos referiremos en este trabajo para dar cuenta del mencionado Epítome, del que se han localizado, hasta la fecha, diversos ejemplares que el anuario Aljamía (2007: 416) enumera y describe con detalle.

La copia del Epítome de la Gramática Arábiga de 1719, redactada por el propio Fr. Bernardino González, ve la luz tras recibir la aprobación de varios profesores de árabe de Tierra Santa y las licencias del Superior de Tierra Santa y de la Orden franciscana. A lo largo de sus 409 páginas incorpora un prólogo y tres tratados diferentes: el primer tratado, que lleva por título De los rudimentos de la lengua arábiga, se divide en cinco capítulos; el tratado segundo, Del verbo arábigo y lo que de él se origina, incluye doce capítulos; y el tercer tratado, titulado Del nombre y partículas, organiza su contenido en cinco capítulos. Estos veintidós capítulos están completados por una recopilación de más de cincuenta temas que tratan cuestiones diversas, entre los que Bueno (2013: 99) señala algunos nombres: "Modo de introducir a hablar", "Copia de nombres y verbos", "Nombres y verbos tocantes a Dios y sus atributos", "Nombres y epítetos de María", "De los ángeles y santos", "De la iglesia así en lo formal como en lo material", "De las festividades", "De los demonios y pecadores", "Del arte militar", "De la mercancía”, "Del gobierno y ministros de justicia" o "De los animales y sabandijas".

Concluimos, tras esta exposición, el apartado dedicado a la vida y obra de Francisco Bernardino González, a quien no podemos sino reconocer, una vez explorada su considerable contribución al campo de la filología árabe de su tiempo, como uno de los más insignes arabistas del siglo XVIII, cuya experiencia en Sevilla sentó las bases de un trabajo que llegaría a culminar en la elaboración de sendas obras que, sin duda, constituyen magníficas piezas lexicográficas y gramaticales que ayudaron a tantos otros interesados en el estudio y conocimiento de la lengua árabe, como bien recoge Bueno (2013: 100) al referir en su artículo que, ambas obras,

4 Véase la página 478.

ISSN $1132-0265$

http://dx.doi.org/10.12795/PH.2017.i31.o1

Philologia Hispalensis 31/1 (2017) 11-36 
fueron los textos que, de forma básica y estable, se utilizaron durante muchos años para enseñar el árabe a los franciscanos españoles que eran enviados a Tierra Santa.

\section{Dos figuras claves: Pascual de Gayangos y León Carbonero y Sol}

Hemos expuesto el decisivo peso que supuso para la ciudad de Sevilla y su Colegio Trilingüe contar con estudiosos de la talla del franciscano Francisco Bernardino González, cuya contribución a las letras árabes lo eleva, por un lado, al rango de figura de primer orden dentro del arabismo español del siglo XVIII, y, por el otro, lo instituye como un referente para otros arabistas que desarrollarían su labor ya en el siglo XIX. Consideramos ahora muy pertinente aludir a la trayectoria vital y las contribuciones de dos personalidades que recogieron la impronta bernardina y dejaron un legado que, sin duda alguna, vino a complementar y enriquecer decisivamente el estudio del árabe no solo en la universidad de capital andaluza, sino en todo el país de forma general. Nos referimos a Pascual de Gayangos y Arce y a León Carbonero y Sol.

\subsection{Pascual de Gayangos}

Podemos considerar a Pascual de Gayangos y Arce como uno de los eruditos más prolíficos de nuestras letras, destacando en las materias de historiografía, numismática, traducción, diplomacia y, especialmente, la bibliografía y el estudio de la lengua árabe. A este respecto, Rocío Velasco de Castro (2009: 250) señala que: "La erudición de Pascual de Gayangos y su intensa labor filológica y de investigación, lo encumbran como el fundador del arabismo español actual, 'el oráculo de su tiempo', y el primero que formó discípulos, logrando que continuaran de manera entusiasta su obra de estudio", palabras que terminan de catalogar a este erudito como una de las figuras más destacadas del panorama arabista en la España de su tiempo.

La conmemoración del bicentenario de su nacimiento nos proporciona abundante documentación sobre la trayectoria vital de este arabista. Gonzalo Anes (2010: 9) nos revela que nació y fue bautizado en Sevilla, el 21 de junio de 1809, en la iglesia parroquial de San Lorenzo, lo que hace a Gayangos hijo de la capital hispalense y origina, de entrada, un primer vínculo geográfico con Sevilla que, como iremos comprobando, tendrá calado posterior en su Universidad. Gayangos procede de una familia de militares, circunstancia que trajo aparejada el desplazamiento de la misma por diferentes puntos de la geografía española y europea, en los que nuestro erudito fue adquiriendo su formación. Así, en 1821 se trasladaba con su familia a Madrid, donde estudió en las Escuelas Pías de los Escolapios y en los Reales Estudios de San Isidro. Al cabo de un año, se desplaza a Francia, donde Gayangos estudia las lenguas francesa, latina y griega, y se inicia en la lengua árabe en la 
École Spéciale des Langues Orientales, bajo la guía del profesor Silvestre de Sacy 5 . En 1830 pasa a trabajar a la Oficina de Interpretación de Lenguas donde recibió el encargo de clasificar e indexar los manuscritos árabes localizados en la Real Biblioteca. En 1834 viaja a Inglaterra y, tal y como señala Rocío Velasco de Castro (2009: 252), empezó a extractary traducir manuscritos árabes del British Museum y publica "Arabic Manuscripts in Spain", en la Westminster Review. En el año 1836 se le concede un puesto como profesor de árabe en el Ateneo de Madrid (Anes 2010: 13). Fue elegido miembro de la Royal Asiatic Society y de la American Academy of Arts and Sciencies en 1841 y 1842, respectivamente, y el año 1843, tras la creación de la cátedra de árabe en la madrileña Universidad de la Corte, Gayangos es nombrado titular de la misma. En los años sucesivos, el arabista sevillano siguió cosechando diferentes galardones y logros que mostraban que se valoraban sus conocimientos de lengua árabe y de historia del Islam, y su formación humanística (Anes 2010: 14), entre ellos la membresía en la Kaiserlichen Akademie des Wissenschaften de Viena y en la Academia Real das Ciencias de Lisboa, en 1844, o en el Institut Imperial de France y la Regia Academia Litterarum Humaniorum Historiarum et Antiquitatum de Suecia, doce años más tarde. Pascual de Gayangos se jubiló como catedrático en 1870. Nueve años más tarde, el día 4 de octubre de 1897, el erudito sevillano fallecía.

Cristina Álvarez Millán nos ofrece en su trabajo una prolija descripción de su obra, conocida en su conjunto como biblioteca oriental de Pascual de Gayangos y catalogada como la tercera colección más importante de manuscritos árabes (2010: 85). La autora realiza una descripción general de la que denomina Colección Gayangos, señalando que no sólo se compone de manuscritos árabes y aljamiados, sino que también está formada por códices en otras lenguas orientales [...] papeles personales, materiales de trabajo y un rico epistolario. El abrumador volumen documental recabado por el bibliófilo sevillano se materializa en un conjunto de valor bibliográfico e historiográfico de primer orden (Álvarez 2010: 85-86). De todo este ingente repertorio, cabe destacar su obra The History of the Mohammedan Dynasties in Spain, la primera fuente histórica que abarcaba la historia de al-Andalus en su totalidady, aún a día de hoy, la única traducción de la obra de al-Maqqari a una lengua de Europa. Los dos volúmenes que componen este trabajo obran en poder de la Biblioteca de Investigadores de la Universidad de Sevilla, e incluyen la traducción de la obra de al-Maqqari del árabe al inglés, acompañada de numerosas notas, apéndices, tablas cronológicas y genealógicas e ilustraciones (Sánchez-Moliní 2001: 62); o las Leyes de Moros del siglo XIV y la Suma de los principales mandamientos y devedamientos de la Ley y Çunna del alfaquí mayor de la aljama de Segovia (Álvarez 2010: 94). La ocupación de la cátedra de árabe en la Universidad de la Corte tuvo más trascendencia de lo que podríamos pensar, pues es a partir de ese momento cuando

\footnotetext{
5 Uno de los más prestigiosos arabistas franceses de todos los tiempos (1758-1838) cuyos estudios gramaticales fueron la base de formación de todos los estudiantes europeos hasta bien entrado el siglo XX.
} 
Gayangos se consolida como el padre del arabismo español, ya que, como señala Cristina Álvarez Millán (2010: 89), la institución madrileña permitió a Gayangos formar varias generaciones de discípulos que habrían de consolidar los estudios árabes en España, los cuales fueron como vástagos que bebieron de su saber y se instruyeron gracias a los volúmenes de su biblioteca personal. Rocío Velasco de Castro (2009: 253-54) nos proporciona la identidad de algunos de ellos: Antonio Machado Álvarez (padre de los afamados literatos Manuel y Antonio Machado), Leopoldo Eguilaz y Yanguas y, muy especialmente, León Carbonero y Sol.

\subsection{León Carbonero y Sol}

León Carbonero, arabista heredero de la erudición gayanguista, constituye el punto de unión de lo hasta ahora expuesto a lo largo del presente apartado con la historia de la enseñanza del árabe en la Universidad de Sevilla. Gonzalo Díaz Díaz (1998: 137-138) nos facilita en su trabajo algunos detalles sobre su persona. Nacido con el nombre de León Carbonero y García-Arisco en la localidad toledana de Villatobas, se dedicó eminentemente a la escritura y al periodismo, y fue un acérrimo defensor y activista católico de su época, lo que le granjeó la concesión del título de Conde de Sol por parte de Pío IX en 1870. Fue enviado a Madrid tras la muerte de su padre, donde estudió tanto en el Colegio Imperial de la Compañía de Jesús de Madrid como en los Reales Estudios de San Isidro, coincidiendo con Gayangos en las lecciones de árabe impartidas por el Padre Artigas. En 1832 comienza a estudiar la carrera de Derecho, doctorándose siete años después. En 1838 recibe la cátedra de árabe de la Universidad de Toledo y, en 1845, tras haber sido discípulo de su antiguo compañero Pascual de Gayangos en el Ateneo de Madrid, ocupaba de forma interina la recién creada cátedra de árabe de la Universidad Literaria de Sevilla, convirtiéndose en el primer catedrático en dicha materia en esta Universidad. Gracias a toda la información que venimos desglosando, podemos reafirmarnos con contundencia en la importancia de la labor de Gayangos para con el desarrollo de lo árabe en la universidad sevillana, pues, a través de su discípulo León Carbonero, la ingente contribución al arabismo español que realizó el insigne maestro pudo permear y enriquecer cualitativamente los estudios que se habrían de emprender en la universidad hispalense ${ }^{6}$. La intensa vida académica de León Carbonero y Sol le retribuyó una posición considerable dentro de la Universidad de Sevilla. El día 19 de junio del año 1846 obtendría la propiedad de la cátedra de árabe, y, tres años después, pasaría igualmente a regentar la cátedra de literatura española. En 1863 ostentó el cargo de Decano de la Facultad, y en 1867 recibía la cátedra del

6 La influencia y preponderancia de ambas figuras parece haber sido tan relevante que la propia página web del Departamento de Filologías Integradas reconoce su contribución a los estudios de árabe mediante la siguiente mención: Las enseñanzas de árabe posteriores a la Ilustración comienzan, como en el resto del país, a la sombra del sevillano Pascual de Gayangos y de sus discípulos en la ciudad. Con el comienzo de la Universidad Literaria de Sevilla en 1845, el primer catedrático de Árabe es León Carbonero y Sol. 
segundo curso de árabe. No obstante, su negativa a jurar la Constitución de 1869 dio lugar a una decisión gubernamental que le acarreó la pérdida de su cátedra de árabe al año siguiente, si bien consiguió acceder de nuevo a la misma en 1873, en calidad de excedente, hasta que se jubiló en 1892.

\section{El ESTUdio DEL ÁRABE EN SEVILla dURANTE LAS ÚlTimaS dÉCADAS}

La perspectiva que hemos recogido y expuesto a lo largo de las diferentes secciones de este trabajo permite ver que el interés por el conocimiento y estudio de la lengua árabe, así como la presencia de instituciones dedicadas al fomento y cultivo de su enseñanza, ha variado ostensiblemente a lo largo de todo el período temporal que hasta ahora hemos considerado, pero la tradición y la impronta arabista consolidada durante siglos en Sevilla se encuentra ya encauzada y amparada en el marco de una institución estable que ofrece a los interesados la formación en lengua y cultura árabes. Nos referimos, como no podía ser de otro modo, a la Universidad de Sevilla tal y como la conocemos entre los siglos XX y XXI.

De entre todas las fuentes oficiales emisoras de documentación a las que podemos recurrir para esclarecer y definir con más precisión el tipo de enfoque en la enseñanza del árabe en nuestra época, los Boletines Oficiales del Estado recogen la evolución diacrónica de los planes de docencia establecidos y la relación de asignaturas dedicadas de manera específica o tangencial a la lengua árabe y su cultura, y que conducen a la obtención de un determinado título universitario. Por lo tanto, dedicaremos este apartado del trabajo a referir y comentar los aspectos más destacados de cada uno de estos planes, y a ver la evolución experimentada por el estudio universitario del árabe desde las etapas más representativas del pasado siglo hasta llegar a la forma en que se imparte en la actualidad.

\subsection{Antecedentes en los planes de estudios}

Los primeros datos que podemos traer a colación con el suficiente rigor científico que aseveren la presencia de una materia dedicada al estudio del árabe en los planes universitarios sevillanos del pasado siglo datan del 22 de julio del año 1955, fecha en la que se publicó en el BOE una orden a día de 4 de julio del mismo mes por la que se aprueba el plan de estudios de la Facultad de Filosofía y Letras de la Universidad de Sevilla.

Con anterioridad a esta fecha, y como consta en la página cuatro de la Memoria para la Solicitud de Verificación del Título Oficial de Graduado o Graduada en Estudios Árabes e Islámicos por la Universidad de Sevilla:

Con el comienzo de la Universidad Literaria de Sevilla en 1845, el primer catedrático de Árabe es León Carbonero y Sol. En 1849 ocupa el puesto no 187 del escalafón, entre los 290 catedráticos de todo el país. En ese momento Pascual de Gayangos ocupa en la Central de Madrid el no 201 y José Moreno Nieto el no 
26o en Granada. Detrás de él se hallan, a finales del XIX, José Vázquez Ruiz, y poco después, Francisco Pagés y Belloc, Rector de la Universidad de Sevilla entre 1900 y 1914. Hasta 1907 solamente se imparte, al igual que en Madrid, Zaragoza y Granada, un curso de árabe clásico en la Facultad de Letras, que en reforma posterior se extiende a Salamanca y Barcelona. Tras años en los que los estudios árabes de Sevilla no cuentan con su correspondiente cátedra, en 1970 se crea la que obtiene Pedro Martínez Montávez.

Deseamos dejar constancia de que la información que hemos llegado a obtener acerca de los estudios árabes entre los años veinte y cincuenta del siglo $\mathrm{XX}$ es escasísima. Nuestras investigaciones en los archivos de la Universidad no han redundado en la obtención de testimonios relevantes, ya que gran parte de la documentación referida a dicho periodo temporal que se conserva en esos fondos se encuentra, en su mayoría, en proceso de tratamiento y, por tanto, no está convenientemente organizada para aportar relevancia a las páginas de este trabajo. Así pues, optamos por contemplar la inclusión de dicha información en estudios posteriores que puedan completar nuestro objeto de estudio, y reconducimos la exposición hacia la descripción del plan de estudios del 22 de julio del año 1955 .

En este plan de estudios se contemplaban dos cursos académicos de formación común y tres cursos de especialización. La lengua árabe contaba entre las materias generales de Filosofía y Letras, y se impartía durante los dos cursos de estudios comunes, en régimen de optatividad con la lengua griega, y con un dedicación de tres horas semanales.

Diez años después, el día 9 de enero de 1965, el BOE hace pública una nueva orden fechada a 11 de diciembre de 1964, que supone el surgimiento de los estudios específicos en Filología, pues se crea en la Facultad de Filosofía y Letras de la Universidad de Sevilla la Sección de "Filología Moderna". Cuatro meses después, el día 22 de mayo de 1965, aparece en el BOE la orden del 21 de abril, por la que se presenta el plan de estudios de la recién creada sección. El año 1968 se revela como especialmente prolífico en cuanto a la diversificación y consolidación de las diferentes ramas o secciones que van surgiendo en la Universidad. Así, se publican sendas órdenes oficiales con fecha de 9 de enero y 17 de octubre, que dan lugar, respectivamente, a la fijación del plan de estudios de la sección de Historia de América, y a la creación de la sección de Arte dentro de la Universidad sevillana. Es de especial interés la segunda de ellas, ya que se incluye específicamente dentro del Plan de Estudios de Arte una asignatura optativa de lengua árabe, que el alumno puede cursar a su discreción en cualquiera de los dos primeros cursos de especialización previstos en la sección.

Durante estos últimos años, la impartición de la docencia correspondiente a la única asignatura de árabe, la denominada "Lengua y literatura árabes" I y II, corre a cargo del profesor Gamal Abdel Karim. 
El profesorado dedicado a la enseñanza de esta materia se verá sucesivamente ampliado con las incorporaciones de otros tres docentes: el profesor Ramón Mendoza Neguillo, que compartirá enseñanza con el anterior a partir del curso 196970; doña Eugenia Gálvez Vázquez, que empieza su actividad en el año académico 1970-71 y, por último, la profesora Clara María Thomas de Antonio, que se une a este grupo en el curso 1973-1974.

Finalmente, el día 16 de enero de 1974 aparece en el BOE una resolución de la Dirección General de Universidades e Investigación, con fecha de 17 de diciembre del año anterior, la cual introduce una reforma en el plan de estudios del primer ciclo de la Facultad de Filosofía y Letras de Sevilla que se traduce en una mayor presencia cuantitativa de la lengua árabe en la sección de Filología.

Esta última etapa coincide con la desaparición del panorama académico de la Universidad de Sevilla de las figuras de Gamal Abdel Karim y Ramón Mendoza, que son sustituidos por los profesores José Vázquez Ruiz y Braulio Justel Calabozo, comenzando así un nuevo periodo cronológicamente ubicado entre los últimos años de la década de los setenta y los primeros de los ochenta.

\subsection{La presencia de la lengua árabe en la Universidad actual y su evolución hasta el siglo XXI}

La última de las modificaciones comentadas se gestó y acaeció, como hemos señalado, entre los años 1973 y 1974, encontrándose recién aprobada en España la llamada Ley General de Educación de 1970 o Ley de "Villar Palasí". Esta ley supuso, en líneas generales, una mayor autonomía para las universidades en lo relativo a impartición de docencia, un impulso de la labor investigadora y un reforzamiento de la figura del departamento. Precisamente el año 1970 nos parece especialmente significativo, ya que, como señala Clara María Thomas de Antonio (2006: 11), nace el Departamento de Árabe de la Universidad de Sevilla de la mano del arabista Pedro Martínez Montávez, quien había obtenido la cátedra de estudios árabes de la Facultad, y cuya labor profesional en la Universidad sentaría las bases para la actual especialidad de Filología Árabe (Thomas 2006: 333).

Con la Ley de Reforma Universitaria (L.R.U.) de 1983 surge, en el año 1985, el Plan de Estudios de la que ya podemos considerar la primera Licenciatura en Filología Árabe de pleno derecho, para la que se estipulan tres cursos de especialización en materia lingüística, literaria e histórica con el elemento árabe como eje central, más dos cursos iniciales, cuyo único requerimiento es incluir en la matrícula las asignaturas de Lengua Árabe I y Lengua Árabe II respectivamente. Este Plan de Estudios se mantuvo durante doce años, hasta que en 1997 se llevó a cabo la última reforma de la licenciatura, que dio lugar a una nueva distribución de asignaturas, y que estuvo vigente hasta principios del siglo XXI. A nuestro parecer, este nuevo y último catálogo de materias supuso el paso definitivo hacia la confección de un título de formación muy específica en materias muy concretas. 
En el año académico 2009-2010, la normativa aplicada en los países pertenecientes al Espacio Europeo de Educación Superior hizo necesario homogeneizar los títulos universitarios a nivel interestatal dentro de la Unión Europea, y sustituir todos los estudios universitarios en vigor por nuevos títulos adaptados a las directrices del Plan Bolonia. De este modo, la Licenciatura en Filología Árabe se convirtió en el llamado Grado en Estudios Árabes e Islámicos.

A esto tenemos que añadir las otras dos titulaciones de nuestra Facultad en las que, sea de manera directa o tangencial, se reserva un papel en su programación curricular para la lengua árabe. Una de ellas es el "Máster Universitario en Traducción e Interculturalidad", que permite a los futuros traductores adquirir maestría en el trasvase de producciones lingüísticas escritas en lengua árabe hacia el español, cuya temática se concreta en las siguientes áreas: textos científico-técnicos y bio-sanitarios, textos jurídico-económicos, textos literarios y textos periodísticos ${ }^{8}$. La otra titulación es el "Máster Universitario en Enseñanza del Español como Lengua Extranjera y Otras Lenguas Modernas", cuyo sexto módulo permite al estudiante entrar en contacto y completar su formación como docente con la incorporación de una segunda lengua, entre ellas el árabe, explorada tangencialmente en dos asignaturas diferentes: Aprendizaje y Adquisición de la Lengua Española por el Alumnado Arabófono e Historia de la Enseñanza de la Lengua Árabe ${ }^{9}$.

Asimismo, no podemos dejar de destacar la importantísima labor llevada a cabo por dos instituciones que trascienden el ámbito universitario y complementan su labor académica ofreciendo cobertura a todos aquellos interesados en el aprendizaje de la lengua árabe. De una parte, el Instituto de Idiomas $^{10}$ de la Universidad de Sevilla, cuyo nacimiento se remonta al año 1927, y en el que los cursos de árabe constituyen una materia consolidada dentro su oferta lingüística, y son impartidos de forma ininterrumpida desde el año académico 1984-1985, tanto a miembros de la Comunidad Universitaria como a alumnos no matriculados en la misma. Y de otra, aludimos también a otro significativo centro de ciencia y conocimiento de la ciudad de Sevilla: la Fundación Tres Culturas ${ }^{11}$. En ella se imparten también, desde su creación, clases de lengua árabe de diferentes niveles dirigidas a toda la población.

Finalmente, dedicamos las últimas líneas de este apartado a referenciar la singular presencia de la lengua árabe en el seno de la Escuela de Comercio de Sevilla. Este centro, que fue creado por el Real Decreto de 11 de agosto de 1887 (Walls

7 Véase Departamento de Filologías Integradas. Estudios árabes e islámicos. <http://departamento. us.es/fintegradas/estudios-arabes-e-islamicos/>.

8 Véase Máster Universitario en Traducción e Interculturalidad. <www.us.es/estudios/master/ master_M112>.

9 Véase Máster Universitario en Enseñanza del Español como Lengua Extranjera y Otras Lenguas Modernas. <www.us.es/estudios/master/master_Mo7o >.

10 Véase Instituto de Idiomas. Universidad de Sevilla. <www. institutodeidiomas.us.es $>$.

${ }^{11}$ Véase Fundación Tres Culturas. <www. tresculturas.org $>$. 
1985: 14) con la finalidad de reorganizar las enseñanzas comerciales, contó, desde la entrada en vigor de las modificaciones planteadas en el Real Decreto de 31 de agosto de 1922, con las asignaturas "Árabe vulgar", en el primer curso del Grado Profesional o Técnico, y “Árabe $2^{\mathrm{o}}$ ”, en el segundo curso del mismo Grado. Ambas asignaturas figuraban en el plan de estudio como optativas junto con los idiomas alemán e italiano (Walls 1985: 87-8). Con las modificaciones aparecidas en la Ley de 17 de julio de 1953 sobre Ordenación de las enseñanzas económicas y comerciales, cuyo plan de estudios fue publicado en el Decreto de 23 de julio del mismo año,

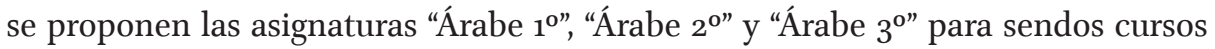
del Grado de Profesor Mercantil, las cuales comparten un régimen de optatividad con las materias de alemán, italiano y portugués (Walls 1985: 125-7). A mediados de los años setenta, a raíz de la reforma educativa en la enseñanza universitaria, este centro pasa a estar incluido en el conjunto de carreras ofrecidas por la Universidad de Sevilla, con la titulación de Escuelas Universitarias de Estudios Empresariales (Walls 1985: 138-40), y, a partir de ese momento, tiene lugar la desaparición de la lengua árabe de sus sucesivos planes de estudio.

\section{Conclusiones}

La realización de este trabajo nos ha permitido comprobar cómo, en el lapso temporal trascurrido entre los casi ocho siglos considerados, la lengua árabe ha sido y sigue siendo objeto de interés y valoración de muy variadas instituciones sevillanas. En efecto, ya con la fundación del Estudio General de Latino y Arábigo a los pocos años de reconquistar Sevilla, vemos una clara muestra de querer conservar, desde el primer momento, el legado dejado por los árabes andalusíes a través del estudio y conocimiento de su lengua, no solo para poder acceder a su abundante producción científico-literaria, sino también como un medio de cohesión social que acercara a dos comunidades que habrían de convivir en el mismo espacio geográfico. No podemos sino calificar de loables las nobles intenciones con las que Alfonso $\mathrm{X}$ estableció el mencionado Estudio General, que vendría a ser la materialización de los primeros pasos dados en pos de ese objetivo y, al mismo tiempo, el punto de partida de la enseñanza del árabe en la ciudad de Sevilla.

De igual modo, comprobamos el enorme peso y trascendencia, tanto a nivel nacional como internacional, que tuvo para el estudio del árabe la actividad intelectual llevada a cabo en el Colegio Trilingüe, pues en sus muros se formaron excepcionales figuras que realizaron aportaciones más que notables al arabismo sevillano y español, y cuya labor marcó las pautas de otros estudiosos posteriores, beneficiarios de su trabajo y contribuidores, igualmente, al desarrollo de las letras árabes en Sevilla y en España entera.

Merece especial reconocimiento y encomio por nuestra parte el celo, afán y profesionalidad con los que los arabistas que hemos traído a colación en nuestro trabajo se entregaron a la redacción de diccionarios y gramáticas para aprender 
y enseñar la lengua. La voluntad manifiesta de llevar a cabo semejante cometido denota el interés y esmero que estos maestros ponían en facilitar a sus pupilos el aprendizaje del árabe, al suministrarles un material elaborado por ellos mismos y que cubriera sus necesidades en la medida de lo posible: el insigne diccionario de Fr. Bernardino y el monumental volumen de textos y manuscritos recogidos por Gayangos y utilizados en sus clases. Todo ello nos permite ver directamente la preocupación de sus autores por adaptar, en la medida de lo posible, los contenidos impartidos, de tal forma que el discente pudiera acceder, con las mejores garantías, al estudio del árabe, y obtener de él el máximo rendimiento.

El enraizamiento en nuestra historia más reciente de esta tradición arabista, bien consolidada y abordada desde los planos intelectual y docente a través de la labor de las personalidades referenciadas, cobra una ulterior relevancia en el devenir del tiempo, que nos permite arrojar una serie de conclusiones que son de aplicación a la situación actual de la enseñanza y transmisión de esta herencia árabe que venimos reflejando. Ha quedado demostrado en la parte final de esta exposición que el interés por la lengua y la cultura árabes no han visto mermada su pujanza en las altas esferas académicas, a saber, en la Universidad y en centros de diversa índole, como son la Fundación Tres Culturas o el Instituto de Idiomas de la Universidad de Sevilla, cuyos planes curriculares ofrecen diversas materias que contemplan el estudio tanto del idioma como de aspectos relativos a la cultura y a la idiosincrasia del pueblo árabe. La dilatada tradición arabista de la ciudad de Sevilla pervive a día de hoy, reflejando los frutos de toda la actividad intelectual forjada en los últimos siglos a través de toda una serie de cursos, asignaturas y planes de estudio, mediante los cuales, variopintos centros de conocimiento que, aunque se ocupan de la instrucción en diferentes ámbitos del conocimiento, convergen en poner todo ello a disposición del discente interesado en una lengua y una cultura vivas, de enorme tradición en la ciudad, y cuyo conocimiento y aprendizaje en la actualidad continúa viéndose fomentado, reforzado y garantizado gracias a la notable labor académica desempeñada dentro de sus muros.

\section{REFERENCIAS BibLIOGRÁFICAS}

Álvarez Millán, Cristina (2010): "El fondo oriental de la Real Academia de la Historia: La Colección Gayangos”, en: Anes y Álvarez de Castrillón, Gonzalo (coord.): Pascual de Gayangos en el bicentenario de su nacimiento. Madrid, 85-106.

ANES y Álvarez de CASTRILlón, Gonzalo (2010): "Don Pascual de Gayangos y Arce en la Real Academia de la Historia”, en: Anes y Álvarez de Castrillón, Gonzalo (coord.): Pascual de Gayangos en el bicentenario de su nacimiento. Pascual de Gayangos en el bicentenario de su nacimiento. Madrid: Real Academia de la Historia, 9-38.

ARANDA, Gabriel de (1698): Vida del siervo de Dios exemplar de sacerdotes el venerable padre Fernando de Contreras natural de esta ciudad de Sevilla. Sevilla: Thomas Lopez de Haro.

Asín PaLacios, Miguel (2007): "El intérprete arábigo de Fr. Bernardino González. Nota bibliográfica”, Boletín de la Real Academia de la Historia 38, 267-279. 
Benito Ruano, Eloy (2000): "Ámbito y ambiente de la Escuela de Traductores de Toledo", Espacio, Tiempo y Forma, Serie III, $H^{a}$ Medieval 13, 13-28.

Benito RuAno, Eloy (2000): Tópicosy realidades de la Edad Media II. Madrid: Real Academia de la Historia..

Bernardino González, Francisco (2005): Intérprete Arábico-Epítome de la Gramática Arábiga [Obras manuscritas]. Edición y estudio preliminar de R. Lourido Díaz. Madrid: Real Academia de la Historia.

Boletín de LA ReAl ACADEMIA de LA Historia (1976). vol. CLXXIII, número III.

BorRero FERNÁNDEZ, Mercedes (1955): Sevilla, ciudad de privilegios: escritura y poder a través del privilegio rodado. Sevilla: Universidad de Sevilla, Servicio de Publicaciones.

Bosch Vilá, Jacinto (1988): Historia de Sevilla. La Sevilla islámica (712-1248). Sevilla: Universidad de Sevilla, Servicio de Publicaciones.

BuENo GARCía, Antonio (2010): "Ética y estética de la traducción monástica. Los traductores y lingüistas franciscanos españoles del árabe y el hebreo", Translationes 2, 1, 25-38.

Bueno García, Antonio (2013): "Las gramáticas y diccionarios de lengua árabe de los franciscanos españoles", Los franciscanos y el contacto de lenguas y culturas, 91-106.

BuRnETT, Charles (1992): "The translating Activity in Medieval Spain”, en: S.Kh. Jayyusi y M. Marín (eds.): The Legacy of Muslim Spain. Leiden-Nueva York-Colonia: Brill, 1036-1058.

BurnetT, Charles (1995): "The Institutional Context of Arabic-Latin Translations of the Middle Age: a Reassessment of the School of Toledo", en: O. Weijers (ed.): Vocabulary of Teaching and Research Between Middle Ages and Renaissance. Turnhout: Brepols, 214-235.

CARrillo SAlCEdo, Juan Antonio (2005): Quinientos años de historia de la Universidad de Sevilla, lección inaugural del aula de la experiencia de la Universidad de Sevilla, curso académico, 2005-2006. Sevilla: Universidad de Sevilla, Secretariado de Publicaciones.

D’Alverny, Marie-Thérèse (1994): La connaisance de l'Islam dans l'Occident Médieval. Aldershot-Brookfield : Routledge.

D’ALVERNY, Marie-Thérèse (1994): La transmission des textes philosophiques et scientifiques au Moyen Âge. Aldershot-Brookfield: Routledge.

Del Castillo Utrilla, María José (1983): "La hospedería de Indias y el atrio del convento casa grande de San Francisco de Sevilla", Actas II Jornadas de Andalucía y América, 393-398.

Del Castillo Utrilla, María José (1998): "El convento de San Francisco, Casa Grande de Sevilla", Arte Hispalense 47, 91-135.

DíAz DíAz, Gonzalo (1998): Hombres y documentos de la filosofía española. Madrid: Consejo Superior de Investigaciones Científicas.

El ImRAni, Abdelouahab (1998): Lexicografía hispano-árabe. Aproximación al análisis de cinco diccionarios elaborados por religiosos españoles. Madrid: Universidad Complutense.

GonZÁLEz FerRín, María Isabel (1999): “Estudio codicológico y diplomático de los tumbos A y B del archivo de la catedral de Sevilla", Historia. Instituciones. Documentos 26, 255-278.

GonzÁLez Jiménez, Manuel (1998): Andalucía a debate. Sevilla: Universidad de Sevilla, Secretariado de Publicaciones.

Haskins, Charles Homer (1924): Studies in the History of Mediaeval Science. Cambridge: Harvard University Press. 
LAdero Quesada, Miguel Angel (1989): Historia de Sevilla. La Ciudad medieval: (1248-1492). Sevilla: Universidad de Sevilla, Servicio de Publicaciones.

LOURIDO DíAz, Ramón (2001): "El conocimiento del árabe entre los franciscanos españoles en Tierra Santa", Archivum Franciscanum Historicum 94, 147-186.

LOURIDO DíAZ, Ramón (2006): "El estudio del árabe entre los franciscanos españoles: Colegio trilingüe de Sevilla-Colegio árabe de Damasco", Archivo Ibero-Americano 66, 9-240.

LoURIDo DíAz, Ramón (2006): El estudio del árabe entre los franciscanos españoles en Tierra Santa: siglos XVII-XIX. Madrid: Cisneros.

Millás Vallicrosa, José María (1933): "El literalismo de los traductores de la corte de Alfonso el Sabio", Al-Andalus 1, 155-187.

Millás VAllicrosa, José María (1942): Las traducciones orientales en los manuscritos de la Biblioteca Catedral de Toledo. Madrid: Instituto Arias Montano.

Millás VAllicrosa, José María (1954): “La corriente de las traducciones científicas de origen oriental hasta fines del siglo XIII", Cahiers d'Histoire Mondiale 2, 2, 395-428.

Procter, Evelyn (1945): "The Scientific Works of the Court of Alfonso X of Castille: the King and his Collaborators", Modern Language Review 40, 12-29.

Procter, Evelyn (1951): Alfonso X of Castille Patron of Literature and Learning. Oxford: Praeger.

SAMsó, Julio (1997): “Traducciones científicas arabo-romances en la Península Ibérica”, en: S. Fortuño, T. Martínez e I. Castellón (eds.): Actes del VII Congrés de l'Associació Hispánica de Literatura Medieval. Vol. 1. Castellón de la Plana: Universitat Jaume I, 199-231.

SÁnChez Herrero, José (1984): "Centros de enseñanza y estudiantes de Sevilla durante los siglos XIII al XV", España medieval 5, 875-898.

SÁnchez-Moliní SÁEz, Carlota (ed.) (2001): La cultura arabo-islámica en la Universidad de Sevilla: Catálogo de fondos impresos de la Biblioteca de Investigadores hasta el año 1900. Sevilla: Grupo de Investigación "El Saber en al-Andalus".

SCARCIA AMORETTI, Bianca Maria (1987): La diffusione delle science islamiche nel Medio Evo Europeo. Roma: Accademia Nazionale dei Lincei.

Thomas de Antonio, Clara María/Giménez Reíllo, Antonio (2006): El saber en AlAndalus, Textos y estudios IV: homenaje al profesor D. Pedro Martínez Montávez. Sevilla: Universidad de Sevilla, Secretariado de Publicaciones.

Thorndike, Lynn/Kibré, Pearl (1963): Catalogue of Incipits of Mediaeval Scientific Writings in Latin. London: Mediaeval Academy of America.

Valderrama, Fernando (OFM) (s.a.): Centuria Bética-Convento y casa grande de San Francisco de Sevilla. Sevilla: Archivo de la Provincia Franciscana Bética (APFB). Fol. 54-55.

Velasco de CASTRo, Rocío (2009): "Arabismo y colonialismo español. Pascual de Gayangos y la cuestión marroquí", Norba, Revista de Historia 22, 245-262.

VERNET, Juan (1978): La cultura hispanoárabe en oriente y occidente. Barcelona: Ariel.

VESPERTINO RoDrígueZ, Antonio (dir.) (2007): Aljamía; anuario de información bibliográfica. Vol. 19. Oviedo: Universidad de Oviedo.

Walls Boza, Federico (1985): La Escuela de Comercio de Sevilla (Compendio Histórico de su vida, desde su creación hasta los umbrales de su centenario). Sevilla: Servicio de Publicaciones del Excmo. Ayuntamiento de Sevilla. 


\section{REFERENCIAS ONLINE}

Departamento de Filologías Integradas. Estudios Árabes e islámicos. < http:// departamento.us.es/fintegradas/estudios-arabes-e-islamicos/> (og marzo 2018).

FUNDACIÓN TRES CULTURAS. <Www. tresculturas.org> (og marzo 2018).

InSTITUTO DE Idiomas. UniversidAd DE SEvilla. <www. institutodeidiomas.us.es> (o9 marzo 2018).

Máster Universitario en EnSeñanza del Español como Lengua Extranjera y Otras LENGUAS MODERNAS. < www.us.es/estudios/master/master_Mo7o> (o9 marzo 2018).

Máster Universitario en Traducción e Interculturalidad. <www.us.es/estudios/ master/master_M112> (o9 marzo 2018).

Pozo RuIz, Alfonso. La primera etapa de la Universidad de Sevilla. <http://personal.us.es/ alporu/historia/historia_1.htm\#hı_2_1> (og marzo 2018).

Universidad de Sevilla. Memoria para la solicitud de verificación del título oficial de Graduado en Estudios Árabes e Islámicos por la Universidad de Sevilla. <webapps.us.es/ fichape/Doc/MV/181_mem Verifica.pdf> (og marzo 2018). 